\title{
Efeito osteoindutor da rosuvastatina, sinvastatina e atorvastatina na Odontologia
}

\author{
Osteoinductive effect of rosuvastatin, simvastatin and atorvastatin in Dentistry \\ Efecto osteoinductor de rosuvastatina, simvastatina y atorvastatina en Odontología
}

Recebido: 22/01/2022 | Revisado: 26/01/2022 | Aceito: 02/02/2022 | Publicado: 04/02/2022

Maria Isabelly Pessoa Brandão ORCID: https://orcid.org/0000-0002-7433-2652 Centro Universitário Maurício de Nassau, Brasil E-mail: brandaoisa12@gmail.com

José Eriverton Sousa Nogueira ORCID: https://orcid.org/0000-0001-8415-8785 Centro Universitário Fametro, Brasil E-mail: erivertonsousa2@gmail.com

Zildenilson da Silva Sousa

ORCID: https://orcid.org/0000-0002-2707-6123 Centro Universitário Maurício de Nassau, Brasil E-mail: Zildenilsonsilva@gmail.com

Matheus Saldanha Botão

ORCID: https://orcid.org/0000-0003-2011-5473 Centro Universitário Maurício de Nassau, Brasil

E-mail: matheussaldanha7@hotmail.com

Shellda Matos de Sousa

ORCID: https://orcid.org/0000-0003-1891-8808 Centro Universitário Fametro, Brasil

E-mail: shelldamatos0192@gmail.com

Manuele Oliveira Moraes

ORCID: https://orcid.org/0000-0001-8823-6605 Centro Universitário Maurício de Nassau, Brasil

E-mail: manumoraeso@gmail.com

José Rafael de Sá Alves

ORCID: https://orcid.org/0000-0002-6124-7563 Universidade Federal do Ceará, Brasil

E-mail: jrafasaalves@alu.ufc.br

Andressa de Sales Fernandes

ORCID: https://orcid.org/0000-0001-7277-5671

Universidade Nove de Julho, Brasil

E-mail: andressasalfer@hotmail.com

Ísis Passos Ferreira

ORCID: https://orcid.org/0000-0001-8540-2954

Centro Universitário Fametro, Brasil

E-mail: isispassosferreira@gmail.com

Julianne Coelho da Silva Cetira

ORCID: https://orcid.org/0000-0002-7004-4848

Universidade Federal do Ceará, Brasil

E-mail: juliannecoelhos@gmail.com

Salma Ivanna Araújo Cavalcante

ORCID: https://orcid.org/0000-0001-9644-5546

Universidade Federal do Ceará, Brasil

Email:salmaaraujo12@hotmail.com

\section{Resumo}

Este estudo de revisão integrativa de caráter exploratório tem como objetivo descrever a eficácia do uso de hipolipemiantes em regeneração óssea alveolar em Odontologia. Para isso, descritores cadastrados no Descritores em Ciências da Saúde (DeCS) e Medical Subject Headings (MeSH) foram aplicadas no Sistema Online de Busca e Análise de Literatura Médica (MEDLINE) por meio do PubMed, além do Google Scholar, Bibliografia Brasileira de Odontologia (BBO) e Literatura Latino-Americana e do Caribe em Ciências da Saúde (LILACS). Foram encontrados inicialmente 1.721 estudos que, após análise minuciosa dos títulos e resumos e avaliação dos critérios de elegibilidade adotados, 17 ensaios clínicos foram incluidos neste estudo. A rosuvastatina, sinvastatina e atorvastatina mostraram-se promissoras no processo de regeneração óssea na periodontia, cirurgia oral e implantodontia, sendo a Índia o país com mais pesquisas neste campo $(n=14)$. Grande parte dos estudos $(n=14)$ avaliaram o efeito dos fármacos associado ao alisamento radicular em periodontite crônica. A concentração de $1,2 \%$ de hipolipemiantes foi a mais frequente nos ensaios selecionados $(n=13)$. Dois estudos evidenciaram valores não significativos de aumento de estrutura óssea quando administrado de maneira isolada. As estatinas demonstram-se como uma terapia promissora em algumas 
especialidades da Odontologia. Todavia, sugere-se que mais estudos na área com amostras maiores e períodos mais extensos sejam realizados visando elucidar melhor o tratamento medicamentoso com tais fármacos na prática odontológica.

Palavras-chave: Regeneração óssea; Proteína morfogenética óssea 2; Hipolipemiantes.

\begin{abstract}
This exploratory integrative review study aims to describe the effectiveness of the use of lipid-lowering agents in alveolar bone regeneration in Dentistry. For this, descriptors registered in Descriptors in Health Sciences (DeCS) and Medical Subject Headings (MeSH) were applied in the Online System of Search and Analysis of Medical Literature (MEDLINE) through PubMed, in addition to Google Scholar, Brazilian Bibliography of Dentistry (BBO) and Latin American and Caribbean Literature in Health Sciences (LILACS). Initially, 1,721 studies were found and, after a thorough analysis of titles and abstracts and evaluation of the eligibility criteria adopted, 17 clinical trials were included in this study. Rosuvastatin, simvastatin and atorvastatin showed promise in the process of bone regeneration in periodontics, oral surgery and implantology, with India being the country with the most research in this field $(\mathrm{n}=$ 14). Most studies $(n=14)$ evaluated the effect of drugs associated with root planing in chronic periodontitis. The concentration of $1.2 \%$ of lipid-lowering agents was the most frequent in the selected trials $(n=13)$. Two studies showed non-significant values of increase in bone structure when administered alone. Statins are a promising therapy in some specialties of dentistry. However, it is suggested that further studies in the area with larger samples and longer periods be carried out in order to better elucidate the drug treatment with such drugs in dental practice.
\end{abstract}

Keywords: Bone regeneration; Bone morphogenetic protein 2; Low lipids.

\title{
Resumen
}

Este estudio exploratorio de revisión integradora tiene como objetivo describir la efectividad del uso de hipolipemiantes en la regeneración ósea alveolar en Odontología. Para ello, se aplicaron descriptores registrados en Descriptors in Health Sciences (DeCS) y Medical Subject Headings (MeSH) en el Sistema en Línea de Búsqueda y Análisis de Literatura Médica (MEDLINE) a través de PubMed, además de Google Scholar, Bibliografía Brasileña de Odontología (BBO) y Literatura Latinoamericana y del Caribe en Ciencias de la Salud (LILACS). Inicialmente, se encontraron 1.721 estudios y, después de un análisis exhaustivo de los títulos y resúmenes y la evaluación de los criterios de elegibilidad adoptados, se incluyeron 17 ensayos clínicos en este estudio. La rosuvastatina, la simvastatina y la atorvastatina se mostraron prometedoras en el proceso de regeneración ósea en periodoncia, cirugía oral e implantología, siendo India el país con más investigación en este campo $(n=14)$. La mayoría de los estudios $(n=14)$ evaluaron el efecto de los fármacos asociados al alisado radicular en la periodontitis crónica. La concentración de $1,2 \%$ de hipolipemiantes fue la más frecuente en los ensayos seleccionados $(n=13)$. Dos estudios mostraron valores no significativos de aumento de la estructura ósea cuando se administró solo. Las estatinas son una terapia prometedora en algunas especialidades de la odontología. Sin embargo, se sugiere que se realicen más estudios en el área con muestras más grandes y períodos más prolongados para dilucidar mejor el tratamiento farmacológico con tales fármacos en la práctica odontológica.

Palabras clave: Regeneración óssea; Proteína morfogenética ósea 2; Lípidos bajos.

\section{Introdução}

Atualmente na Odontologia, há uma grande necessidade de identificar materiais que visem a indução e a formação óssea alveolar (Souza et al., 2016), promovendo uma melhor resposta nos parâmetros clínicos nos pacientes (Yaghobee et al., 2020). Técnicas inovadoras e minimamente invasivas tem sido implementada visando uma reabilitação oral mais rápida (Chauhan et al., 2014), além de possibilitar uma melhor qualidade de vida aos indivíduos que buscam tratamento odontológico (Anbinder et al.,2006). Alguns procedimentos, relacionados à cirurgia e a implantodontia, além de doenças como periodontite, podem gerar danos extensos ao tecido ósseo. Esse tecido possui em sua formação a capacidade de regeneração sem evidenciar vestígios do processo traumático pós procedimentos invasivos na região avaliada, porém quando esta lesão possuir grandes dimensões poderá resultar em um tecido conjuntivo fibroso, o que pode prejudicar o efeito de consolidação óssea (Oliveira et al., 2015).

Visando reduzir esse efeito, um material que tem sido amplamente investigado na Odontologia nos últimos anos devido seu efeito osteocondutor são as estatinas (Souza et al., 2016; Souza et al., 2017; Chauhan et al., 2014; Oliveira et al., 2015; Rao et al., 2013). Pertencentes a classe de hipolipemiantes, estudos revelam que tais medicamentos podem ser uma alternativa eficaz na promoção de regeneração óssea (Silva et al., 2008; Rao et al., 2013; Oliveira et al., 2015; Oliveira et al., 
2017). As estatinas são fármacos que possuem a capacidade de inibição da enzima 3-hidroxi-3-metilglutaril-coenzima A redutase (HMG-CoA), gerando um aumento da expressão do gene da proteína morfogenética óssea - 2 (BMP-2), importante fator de crescimento do tecido ósseo (Chauhan et al., 2014; Souza et al., 2016).

Quanto ao seu processo osteoindutor, essa proteína age como fator de crescimento multifuncional que realiza a regeneração de tecido ósseo (Anbinder et al., 2006; Oliveira et al., 2017; Chauhan et al., 2014) por meio de cadeias laterais que se ligam ao sítio ativo da enzima e bloqueiam o estado de modificação do substrato-produto (Chauhan et al., 2014). Tais ações no organismo possuem a capacidade de promover melhorias nos parâmetros clínicos de patologias frequentemente encontradas na prática odontológica, como por exemplo a periodontite crônica (Souza et al., 2016). Diante deste fato, pesquisadores passaram a realizar estudos relacionados à utilização dessa terapia na cavidade oral após procedimentos odontológicos que resultam em grande perda óssea (Claudio et al., 2017)

Os defeitos ósseos que ocorrem após alguns procedimentos orais são preocupantes na Odontologia porque podem causar graves problemas estéticos e funcionais aos pacientes (Chauhan et al., 2014; Oliveira et al., 2015). Com relação aos efeitos das estatinas, é possível observar um aumento da expressão de BMP-2 (Anbinder et al., 2006), um potente simulador da diferenciação dos osteoblastos e de sua atividade, e elas promovem a mineralização pelos osteoblastos, evidenciado por meio de estudos em cultura celular, indicando que as estatinas têm um efeito anabólico no osso (Horiuchi \& Maeda, 2006).

Com isso, as estatinas, além de pertencerem a classe de hipolipemiantes possuem propriedades que podem promover respostas promissoras no tratamento odontológico por meio da estimulação da BMP-2 (Oliveira et al., 2015; Anbinder et al., 2006). Parece plausível citar que as estatinas podem ser protetoras não apenas contra doenças cardiovasculares, mas também no tratamento de doenças orais, agindo de maneira anti-inflamatória (Rao et al., 2013). Todavia, para Gutierrez et al. (2006) tais fármacos ao serem administrados em doses hipolipemiantes, provavelmente não atingirão concentrações sanguíneas suficientes para causar, de forma confiável, aumentos substanciais na formação óssea em humanos.

Com base nos dados supracitados, o objetivo do presente estudo foi realizar uma revisão integrativa sobre o uso de hipolipemiantes em regeneração óssea em procedimentos odontológicos, visando descrever sua eficácia de maneira isolada ou associada a outros fármacos avaliados em ensaios clínicos randomizados ou não randomizados.

\section{Metodologia}

\subsection{Estratégia de pesquisa}

Trata-se de um artigo de revisão integrativa realizado no mês de maio a setembro de 2021. Sobre este tipo de estudo, Ercole et al. (2014) destacam que visa unir determinados resultados obtidos por estudos já publicados na literatura científica sobre uma determinada temática com o objetivo de sintetizar e obter um maior número de dados para posterior discussão e atualizações sobre a problemática avaliada.

Desse modo, para a idealização, seguiu-se as 6 etapas operacionais descritas por Souza; Silva e Carvalho (2010): 1) Elaboração da pergunta norte da pesquisa, 2) Busca na literatura através de mecanismos metodológicos previamente elaborado pelos autores, 3) Coleta das amostragens obtidas nas bases de dados, 4) Análise minuciosa dos estudos visando sua inclusão e exclusão, 5) Discussão dos resultados obtidos e 6) Apresentação da revisão integrativa.

Após isso, utilizou-se do acrônimo PICOS ( $\mathrm{P}$ = população, $\mathrm{I}=$ intervenção, $\mathrm{C}=$ controle, $\mathrm{O}=$ desfecho e $\mathrm{S}=$ desenho do estudo) (Santos; Pimenta E Nobre, 2007). Com base no acrônimo utilizado, a seguinte pergunta norteadora foi idealizada para guiar a presente revisão integrativa: "O uso de hipolipemiantes é eficaz no processo de regeneração óssea alveolar após procedimentos odontológicos com perda desse tecido conjuntivo especializado?"

Tendo esta pergunta idealizada pelos autores, os descritores cadastrados nos Descritores em Ciências da Saúde (DeCS) e na Medical Subject Headings (MeSH) "Dentistry", "Hypolipidemic Agents/Hipolipemiantes”, "Bone Regeneration/ 
Regeneração Óssea”, "Dentistry/ Odontologia”, “Clinical Trial/ Ensaio Clínico” e "Randomized Controlled Trial/ Ensaio Clínico Controlado Aleatório" foram aplicados no Sistema Online de Busca e Análise de Literatura Médica (MEDLINE) através do PubMed, além do Google Scholar, Literatura Latino-Americana e do Caribe em Ciências da Saúde (LILACS) e Biografia Brasileira de Odontologia (BBO) por intermédio da Biblioteca Virtual em Saúde (BVS). Os descritores foram interligados através dos operadores booleanos "AND" e "OR", visando obter dados científicos relacionados à temática em questão, conforme destacado no Quadro 1 e 2.

Quadro 1 - Acrônimo PICOS da revisão integrativa.

\begin{tabular}{|c|c|c|}
\hline Acrônimo & Sigla & Definição \\
\hline Paciente & $\mathbf{P}$ & $\begin{array}{l}\text { Pacientes em procedimentos odontológicos } \\
\text { "Odontologia/ Dentistry" }\end{array}$ \\
\hline Intervenção & $\mathbf{I}$ & $\begin{array}{c}\text { Medicamentos Hipolipemiantes } \\
\text { "Hipolipemiantes/ Hypolipidemic Agents" } \\
\text { "Inibidores de Hidroximetilglutaril-CoA Redutases/ } \\
\text { ydroxymethylglutaryl-CoA Reductase Inhibitors" }\end{array}$ \\
\hline Controle ou comparação & $\mathbf{C}$ & Não utilizado \\
\hline Desfecho & $\mathbf{O}$ & $\begin{array}{c}\text { Regeneração no tecido ósseo } \\
\text { "Regeneração Óssea/ Bone Regeneration" }\end{array}$ \\
\hline Desenho do estudo & $\mathbf{S}$ & $\begin{array}{c}\text { Ensaios clínicos randomizados ou não } \\
\text { "Clinical Trial", "Randomized Controlled Trial" }\end{array}$ \\
\hline
\end{tabular}

Fonte: Autores (2021), com base em Santos; Pimenta e Nobre (2007).

Quadro 2 - Estratégia de busca na literatura por meio dos operadores booleanos e descritores cadastrados do DeCS e MeSH.

\begin{tabular}{|c|c|c|c|c|}
\hline $\begin{array}{c}\text { Base da } \\
\text { dados }\end{array}$ & PubMed/Medline & LILACS & Google Scholar & BBO \\
\hline Estratégia de & Hypolipidemic Agents & Regeneração Óssea & Hypolipidemic Agents & Regeneração Óssea \\
busca & AND Bone Regeneration & AND Inibidores de & AND Bone Regeneration & AND Inibidores de \\
& AND Dentistry AND & Hidroximetilglutaril- & AND Dentistry AND & Hidroximetilglutaril- \\
& Clinical Trial OR & CoA Redutases OR & Randomized Controlled & CoA Redutases OR \\
& Randomized Controlled & Dentistry & Trial & Dentistry \\
& Trial & & & \\
\hline
\end{tabular}

Fonte: Autores (2021).

\subsection{Critérios de elegibilidade}

Visando obter uma maior confiabilidade científica dos dados coletados, neste estudo apenas ensaios clínicos randomizados ou não randomizados, publicados nos últimos 10 anos (2011 a 2021), sem distinção de idioma e que avaliavam o efeito de hipolipemiantes em regeneração óssea na Odontologia foram classificados para os critérios de inclusão. Os estudos deveriam ter em seus grupos testes pelo menos um dos seguintes fármacos: rosuvastatina, sinvastatina e atorvastatina.

Estudos de revisão, relatos de caso e experiência, teses e dissertações, anais de eventos, documentos editoriais, carta para o editor, livros, capítulos de livros, ensaios pré-clínicos, estudos pilotos, estudos in vitro e in vivo, trabalhos de conclusão de curso (TCC), estudos repetidos, indisponíveis e em outros campos das ciências da saúde com a classe de fármacos em questão que não fossem na Odontologia foram removidos desta revisão.

Com base nesses critérios definidos, 1.411 estudos na soma total das 4 estratégias de busca foram localizados (Figura 1). Destes estudos, 20 foram removidos por itens duplicados nas bases e bibliotecas consultadas. Após análise minuciosa dos títulos e resumos, bem como a leitura completa do estudo na íntegra, avaliando todos os critérios de elegibilidade adotados, 17 ensaios clínicos foram selecionados.

Assim, na primeira estratégia de pesquisa através da MEDLINE/PubMed obteve-se 09 dados que após análise e leitura dos estudos todos foram incluídos. Na BBO e LILACS encontrou-se 01 estudo em cada base, que posteriormente após análise apenas 01 ensaio clínico foi selecionado. Na terceira estratégia de pesquisa, a literatura cinzenta foi utilizada (Google 
Scholar), sendo encontrados 1.710 estudos que após remoção de duplicatas e avaliando os critérios de elegibilidade adotados, 07 estudos foram incluídos.

\subsection{Análise quantitativa e qualitativa dos dados obtidos}

O software Mendeley Desktop 1.19.8 foi utilizado para remoção de referências duplicadas. Quanto ao nível de evidências dos estudos, apenas ensaios clínicos foram selecionados visto o seu alto nível de confiabilidade científica. Para avaliar tais informações, seguiu-se o nível de evidências, descrito pela agência americana Agency for Healthcare and Research and Quality $(A H R Q)$, sendo que todos os estudos incluídos possuíam nível 2 para ensaios clínicos com randomização ou nível 3 para ensaios sem randomização (Stillwell et al., 2010).

Todas as amostras quantitativas e qualitativas foram tabuladas na Tabela 1 através do software Microsoft Excel 2013, sendo destacadas informações como o nome e ano do estudo, amostras totais, país do estudo, materiais avaliados, e desfecho dos ensaios clínicos selecionados. O mesmo software foi utilizado para a elaboração dos Gráficos 1 e 2 contendo as amostras e porcentagens mais prevalentes na literatura, além do fluxograma da pesquisa (Figura 1), conforme destacado abaixo.

Figura 1 - Fluxograma PRISMA (adaptado) da estratégia de pesquisa adotada no estudo.

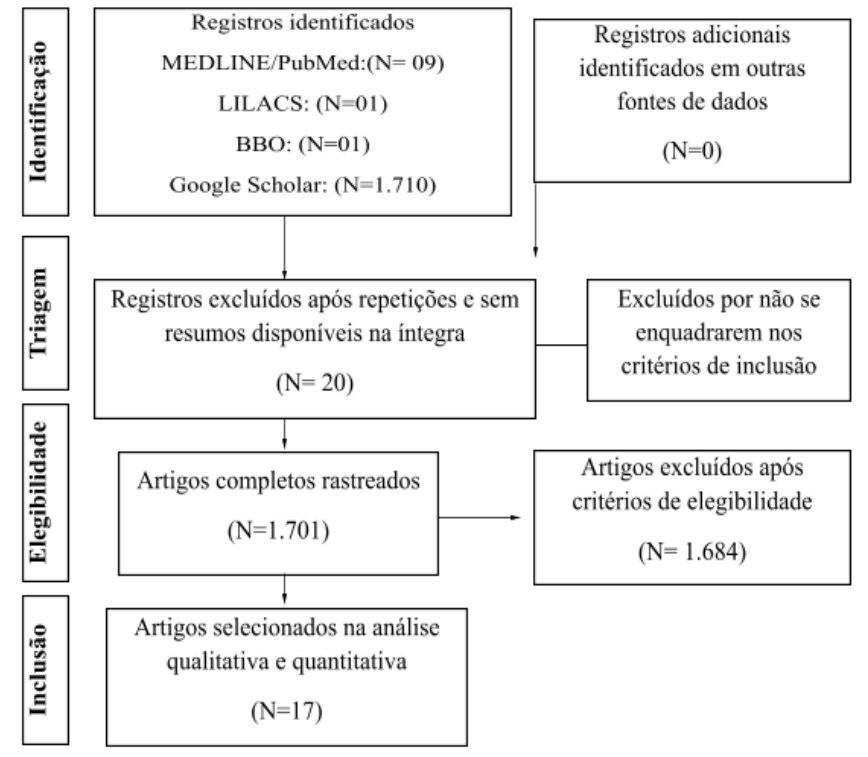

Fonte: Autores (2021), com base no fluxograma PRISMA, descrito por Galvão et al. (2015).

\section{Resultados}

Os 17 estudos selecionados obtiveram uma amostragem total de 1.191 participantes. Os critérios de inclusão de pacientes nos ensaios clínicos em 16 estudos selecionados eram pessoas sistemicamente saudáveis, descritos como ASA I conforme classificado pela American Society of Anesthesiologists (ASA), com idade mínima de 16 e máxima de 55 anos. Os critérios de exclusão adotados pelos autores nestes 16 ensaios clínicos foram: histórico de alergia a estatinas, terapia com estatinas, qualquer condição sistêmica ou medicamento que altera a condição no tratamento odontológico, pacientes imunocomprometidos, com distúrbios hematológicos, presença de periodontite agressiva, uso de substância químicas como o tabaco, além de mulheres grávidas e lactantes.

Apenas um estudo (ensaio clínico controlado randomizado de Kumari et al., 2016) avaliou o efeito regenerativo ósseo em 75 pacientes com periodontite crônica e classificados como ASA II, os mesmos possuíam diabetes melitus do tipo 2, sendo que destes 38 eram homens e 37 mulheres de faixa etária de 40 a 50, no qual também evidenciou desfechos positivos da 
atorvastatina a 1,2\% em defeitos intraósseos. Assim, os hipolipemiantes mais presentes na literatura foram a sinvastatina presente em 08 estudos (42\%), seguida da rosuvastatina em 06 (32\%) e da atorvastatina em 05 ensaios clínicos selecionados (26\%), conforme destacado no Gráfico 1.

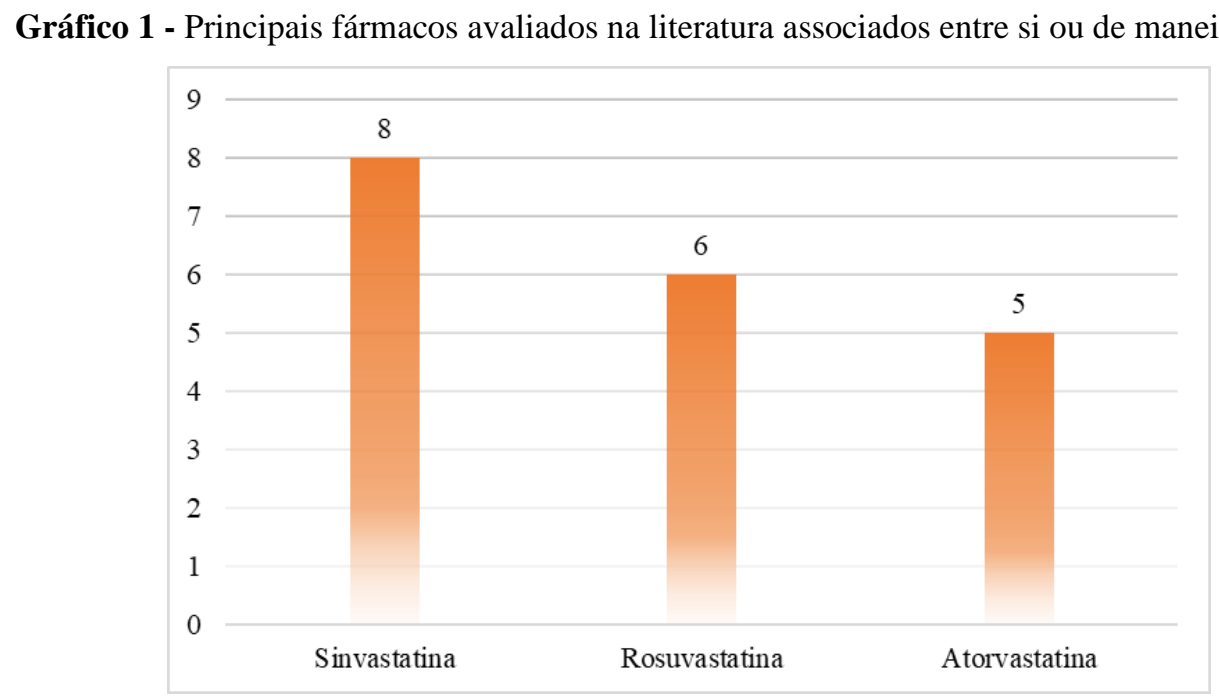

Fonte: Autores (2021), por meio do software Microsoft Excel 2013.

Tais fármacos foram divididos em subgrupos testes e controles, dos quais foram avaliados em associação ao álcool e etanol para diluição nas substâncias em pó sob porcentagens variáveis entre $70 \%$ e $97 \%$. Apenas dois estudos demonstraram efeitos estatisticamente não significativos em seu estudo com as estatinas por meio da avaliação das amostras após procedimento em um período de 06 a 09 meses de avaliação. O estudo de Yaghobee et al. (2020) na área da implantodontia demonstrou que a utilização da sinvastatina foi ineficaz no ganho de tecido ósseo na região do seio maxilar. Esse resultado foi evidenciado por meio da utilização da análise histológica e histomorfométrica obtida de 93 participantes de sexo não especificado no estudo.

Com resultados similares, o ensaio clínico de Martande et al. (2016) demonstrou que a concentração de 1,2\% da atorvastatina não aumentou o potencial regenerativo de fibrina rica em plaquetas (FRP) quando administrado sozinho em defeitos intraósseos na periodontite crônica de 96 participantes. Contudo, a atorvastatina administrada em associação ao FRP evidenciou melhorias semelhantes nos parâmetros clínicos com uma maior porcentagem de redução da profundidade do defeito, visto por meio de radiografias. Além desses achados, materiais complementares foram utilizados em associação ou comparação às estatinas visando demonstrar sua aplicabilidade clínica. Com isso, membranas de barreiras perfuradas, hidroxiapatita porosa, utilização de álcool, ácido etilenodiamino tetra-acético ou etanol foram usados para diluição dos fármacos e aplicados em defeitos intraósseos de pacientes em alguns estudos (Isssa et al., 2020; Yaghobee et al., 2020; Gouda et al., 2021).

Visando ilustrar melhor os dados coletados, optou-se por compilar os principais achados dos estudos na Tabela 1 por meio da ferramenta software Microsoft Excel 2013. A tabela demonstra de maneira resumida os principais achados na literatura científica sobre a temática em questão como os autores, ano de publicação, dados qualitativos e quantitativos como o total da amostra obtida em cada estudo, país em que foi realizado tais pesquisas, classificação do nível de evidências, campo de estudo avaliado, fármacos e dosagens utilizadas e o desfecho do ensaio clínico. 
Tabela 1 - Compilação dos principais dados dos estudos selecionados.

\begin{tabular}{|c|c|c|c|c|}
\hline $\begin{array}{l}\text { Autor/Ano de } \\
\text { publicação }\end{array}$ & $\begin{array}{c}\text { Total da amostra/País } \\
\text { do estudo/Nível de } \\
\text { evidência }\end{array}$ & $\begin{array}{l}\text { Campo de estudo } \\
\text { da Odontologia }\end{array}$ & $\begin{array}{c}\text { Materiais avaliados e } \\
\text { posologia nos ensaios clínicos }\end{array}$ & Desfecho dos estudos \\
\hline $\begin{array}{l}\text { Gouda et al. } \\
\qquad(2021)\end{array}$ & $\begin{array}{c}\text { N=20 (Gênero não } \\
\text { especificado) } \\
\text { País: Egito } \\
\text { Nível de evidência: } 2\end{array}$ & Implantodontia & $\begin{array}{c}\text {-50 mg de Puerarina } \\
\text { - Pó de Sinvastatina dissolvido } \\
\text { em } 97 \% \text { de etanol }\end{array}$ & $\begin{array}{l}\text { Puerarina e Sinvastatina são } \\
\text { materiais de enxerto ósseo } \\
\text { clinicamente benéficos e seguros } \\
\text { no processo de ganho ósseo. }\end{array}$ \\
\hline $\begin{array}{l}\text { Issa et al. } \\
\quad(2020)\end{array}$ & $\begin{array}{l}\text { N=40 (12 homens e } 28 \\
\text { mulheres) } \\
\text { País: Egito } \\
\text { Nível de evidência: } 2\end{array}$ & Periodontia & $\begin{array}{c}\text { - Gel de sinvastatina } \\
\text { - Gel de EDTA } \\
\text { - Membranas de barreira } \\
\text { perfuradas }\end{array}$ & $\begin{array}{l}\text { PBM em associação com SMV } \\
\text { melhora os parâmetros clínicos } \\
\text { do tecido duro em comparação } \\
\text { com as membranas oclusivas no } \\
\text { tratamento de defeitos } \\
\text { periodontais intraósseos. }\end{array}$ \\
\hline $\begin{array}{l}\text { Yaghobee } e t \\
\text { al. }(2020)\end{array}$ & $\begin{array}{c}\text { N=93 (Gênero não } \\
\text { especificado) } \\
\text { País: Irão } \\
\text { Nível de evidência: } 2\end{array}$ & Implantodontia & $\begin{array}{l}\text { - Sinvastatina } 100 \mathrm{ml} \\
\text { dissolvidas em } 70 \% \text { de etanol } \\
\text { - } 8,33 \mathrm{mg} \text { Sinvastatina }\end{array}$ & $\begin{array}{l}\text { Não houve efeito positivo } \\
\text { significativo para a utilização de } \\
\text { sinvastatina no aumento do nível } \\
\text { ósseo do seio maxilar. }\end{array}$ \\
\hline $\begin{array}{l}\text { Chatterjee } \text { et } \\
\text { al. }(2019)\end{array}$ & $\begin{array}{c}\text { N=100 (47 homens e } 53 \\
\text { mulheres) } \\
\text { País: Índia } \\
\text { Nível de evidência: } 2\end{array}$ & Periodontia & $1,2 \%$ de ruvostatina & $\begin{array}{l}1,2 \% \text { administrado localmente } \\
\text { tem efeito anabólico e anti- } \\
\text { inflamatório ósseo e pode } \\
\text { emergir como um agente } \\
\text { modulador do hospedeiro }\end{array}$ \\
\hline $\begin{array}{l}\text { Pooja et al. } \\
\quad(2019)\end{array}$ & $\begin{array}{l}\mathrm{N}=30 \text { (14 homens e } 16 \\
\text { mulheres) } \\
\text { País: Índia } \\
\text { Nível de evidência: } 2\end{array}$ & Periodontia & $1,2 \%$ de sinvastatina & $\begin{array}{l}\text { Maior redução na DP, mais } \\
\text { ganho de CAL e maior } \\
\text { preenchimento de defeito } \\
\text { intraósseo em locais tratados com } \\
\text { SRP e sinvastatina do que SRP e } \\
\text { placebo isoladamente. }\end{array}$ \\
\hline $\begin{array}{l}\text { Shirke et al. } \\
\quad(2019)\end{array}$ & $\begin{array}{l}\text { N=20 (10 homens e } 10 \\
\text { mulheres) } \\
\text { País: Índia } \\
\text { Nível de evidência: } 2\end{array}$ & Periodontia & $1,2 \%$ de atorvastatina & $\begin{array}{l}\text { ATV, associado a SRP, melhora } \\
\text { a regeneração periodontal. }\end{array}$ \\
\hline $\begin{array}{c}\text { Degala \& } \\
\text { Bathija. (2018) }\end{array}$ & $\begin{array}{l}\text { N=30 (Gênero não } \\
\text { especificado) } \\
\text { País: Índia } \\
\text { Nível de evidência: } 2\end{array}$ & Cirurgia Oral & $\begin{array}{c}\text { - } 10 \text { mg de sinvastatina } \\
\text { - Gelfoam }\end{array}$ & $\begin{array}{l}\text { Aplicação local de sinvastatina } \\
\text { pode ser uma forma econômica e } \\
\text { simples de estimular e acelerar a } \\
\text { regeneração óssea. }\end{array}$ \\
\hline $\begin{array}{l}\text { Pankaj et al. } \\
\quad(2018)\end{array}$ & $\begin{array}{l}\text { N=90 (44 homens e } 46 \\
\text { mulheres) } \\
\text { País: Índia } \\
\text { Nível de evidência: } 2\end{array}$ & Periodontia & $\begin{array}{l}-1,2 \% \text { de rosuvastatina } \\
-1 \% \text { de gel de metformina }\end{array}$ & $\begin{array}{l}\text { Os resultados foram } \\
\text { significativamente melhores com } \\
\text { o uso de gel de RSV a } 1,2 \% \text { do } \\
\text { que gel de } \mathrm{MF} \text { a } 1 \% \text {. }\end{array}$ \\
\hline $\begin{array}{l}\text { Garg \& } \\
\text { Pradeep. } \\
(2017)\end{array}$ & $\begin{array}{c}\text { N=105 (55 homens e } 60 \\
\text { mulheres) } \\
\text { País: Índia } \\
\text { Nível de evidência: } 2\end{array}$ & Periodontia & $\begin{array}{l}-1,2 \% \text { de rosuvastatina } \\
-1,2 \% \text { de atorvastatina }\end{array}$ & $\begin{array}{l}\text { RSV mostra melhora } \\
\text { significativa em todos os } \\
\text { parâmetros clínicos. }\end{array}$ \\
\hline $\begin{array}{l}\text { Martande } e t \\
\text { al. }(2017)\end{array}$ & $\begin{array}{l}\text { N=96 (50 homens e } 46 \\
\text { mulheres) } \\
\text { País: Índia } \\
\text { Nível de evidência: } 2\end{array}$ & Periodontia & $\begin{array}{l}-1,2 \% \text { de atorvastatina } \\
-1,2 \% \text { de sinvastatina }\end{array}$ & $\begin{array}{l}\text { ATV resultou em maiores } \\
\text { melhorias nos parâmetros } \\
\text { clínicos da periodontia. }\end{array}$ \\
\hline $\begin{array}{l}\text { N-Prianka et } \\
\text { al. }(2017)\end{array}$ & $\begin{array}{l}\text { N=24 (14 homens e } 10 \\
\text { mulheres) } \\
\text { País: Índia } \\
\text { Nível de evidência: } 2\end{array}$ & Periodontia & $1,2 \%$ de sinvastatina & $\begin{array}{l}\text { Sinvastatina forneceu um método } \\
\text { confortável para melhorar os } \\
\text { parâmetros clínicos e promover a } \\
\text { neoformação óssea. }\end{array}$ \\
\hline $\begin{array}{l}\text { Kumari et al. } \\
\text { (2016) }\end{array}$ & $\begin{array}{l}\text { N=75 (38 homens e } 37 \\
\text { mulheres) } \\
\text { País: Índia } \\
\text { Nível de evidência: } 2\end{array}$ & Periodontia & $1,2 \%$ de atorvastatina & $\begin{array}{l}\text { ATV administrada localmente foi } \\
\text { considerada eficaz no tratamento } \\
\text { de defeitos intraósseos em CP em } \\
\text { pacientes com DM2. }\end{array}$ \\
\hline $\begin{array}{l}\text { Martande } e t \\
\text { al. }(2016)\end{array}$ & $\begin{array}{l}\text { N=96 (48 homens e } 48 \\
\text { mulheres) } \\
\text { País: Índia } \\
\text { Nível de evidência: } 2\end{array}$ & Periodontia & $\begin{array}{c}- \text { PRF } \\
-1,2 \% \text { de gel de atorvastatina }\end{array}$ & $\begin{array}{l}1,2 \% \text { ATV não obteve sucesso } \\
\text { clínico no aumento do potencial } \\
\text { regenerativo de PRF sozinho em } \\
\text { doenças periodontais. }\end{array}$ \\
\hline
\end{tabular}




\begin{tabular}{|c|c|c|c|c|}
\hline $\begin{array}{l}\text { Pradeep et al. } \\
\quad(2016 \mathrm{a})\end{array}$ & $\begin{array}{c}\text { N=90 (45 homens e } 45 \\
\text { Mulheres) } \\
\text { País: Índia } \\
\text { Nível de evidência: } 2\end{array}$ & Periodontia & $\begin{array}{c}-1,2 \% \text { de rosuvastatina } \\
-1,2 \% \text { de atorvastatina em gel }\end{array}$ & $\begin{array}{l}\text { A aplicação local de drogas de } \\
1,2 \% \text { RSV resulta em melhora } \\
\text { clínico-radiográfica } \\
\text { significativamente maior do que } \\
1,2 \% \text { ATV. }\end{array}$ \\
\hline $\begin{array}{l}\text { Pradeep et al. } \\
\quad(2016 \mathrm{~b})\end{array}$ & $\begin{array}{l}\text { N=90 (Gênero não } \\
\text { especificado) } \\
\text { País: Índia } \\
\text { Nível de evidência: } 2\end{array}$ & Periodontia & $\begin{array}{l}\text { - } 1,2 \% \text { de rosuvastatina } \\
\text { - Fibrina rica em plaquetas }\end{array}$ & $\begin{array}{l}\text { Desbridamento com retalho } \\
\text { aberto (OFD) com RSV a } 1,2 \% \text { e } \\
\text { FRP resultou em benefícios } \\
\text { periodontais significativamente } \\
\text { maiores em comparação com } \\
\text { OFD sozinho ou com FRP. }\end{array}$ \\
\hline $\begin{array}{l}\text { Pradeep et al. } \\
\quad(2016 \mathrm{c})\end{array}$ & $\begin{array}{c}\text { N=110 (60 homens e } 50 \\
\text { mulheres) } \\
\text { País: Índia } \\
\text { Nível de evidência: } 2\end{array}$ & Periodontia & $\begin{array}{c}\text { - OFD + gel placebo } \\
\text { - PRF + HA com OFD } \\
-1,2 \% \text { de rosuvastatina em gel } \\
+ \text { PRF + HA com OFD }\end{array}$ & $\begin{array}{l}\text { Combinação de RSV, PRF e HA } \\
\text { tem efeitos sinérgicos, } \\
\text { explicando seu papel como } \\
\text { material regenerativo no } \\
\text { tratamento de defeitos de furca. }\end{array}$ \\
\hline $\begin{array}{l}\text { Pradeep et al. } \\
\quad \text { (2012) }\end{array}$ & $\begin{array}{c}\text { N=72 (38 homens e } 34 \\
\text { mulheres) } \\
\text { País: Índia } \\
\text { Nível de evidência: } 2\end{array}$ & Periodontia & - $1,2 \%$ de de sinvastatina & $\begin{array}{l}\text { Sinvastatina fornece um método } \\
\text { confortável e flexível para } \\
\text { melhorar os parâmetros clínicos e } \\
\text { para aumentar a formação óssea. }\end{array}$ \\
\hline
\end{tabular}

Atorvastatina em gel (ATV); Rosuvastatina (RSV); Membranas de barreira perfuradas (PBM); Gel de sinvastatina (SMV); Fibrina rica em plaquetas (PRF); hidroxiapatita porosa (HA); desbridamento de retalho aberto (OFD); diabetes mellitus tipo 2 (t2DM) / (DM2); raspagem e alisamento radicular (SRP); hidroxiapatita porosa (HA); nível de adesão clínica (CAL); Metformina (MF).

Fonte: Autores, com base nos dados obtidos (2021).

Com relação ao campo de estudo, a maioria estava relacionada a periodontite crônica, com um total de 14 estudos (82\% da amostra total), seguido da implantodontia (12\%) e com uma menor prevalência a cirurgia oral com apenas 1 estudo incluído (6\%), conforme ilustrado no Gráfico 2. Quanto aos locais de estudo, o país em que mais realizou testes com o fármaco foi a Índia com um total de 14 estudos, seguida do Egito com 2 estudos. Com uma menor prevalência o Irã com apenas 1 estudo selecionado de acordo com os critérios de elegibilidade adotados nesta presente revisão.

Gráfico 2 - Desenhos dos estudos mais presentes na literatura seguido do país.

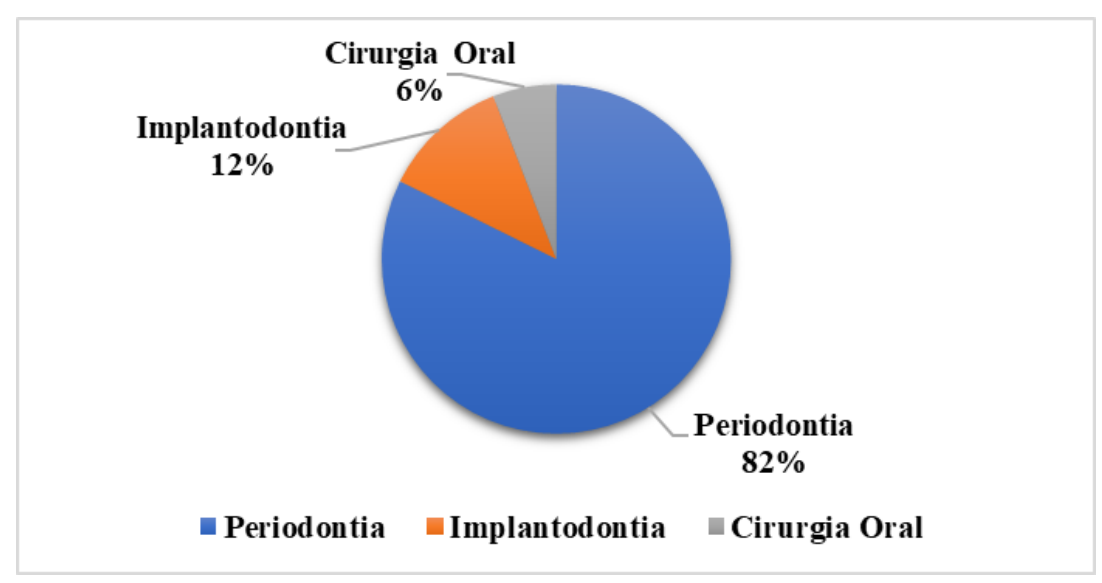

Fonte: Autores (2021), elaborado por meio do software Microsoft Excel 2013.

Quanto ao ano de publicação dos estudos, observou-se que houve uma prevalência maior no ano de 2016 pela temática em questão com a presença de 4 estudos no campo da periodontia publicados na Índia, dos quais 3 demonstraram efeitos promissores da sinvastatina a 1,2\% em associação ou não a fibrina rica em plaqueta. Com base nos dados, evidenciouse que tais fármacos tiveram um declínio de ensaios clínicos publicados a partir do ano de 2020. Quanto aos demais dados qualitativos nos 17 ensaios selecionados, 4 estudos (Pradeep et al., 2016b; Degala \& Bathija, 2018; Yaghobee et al., 2020 e Gouda et al., 2021) não relataram o gênero dos participantes da pesquisa. Com base nas amostras coletadas e evidenciadas nos ensaios clínicos, observou-se que a maioria eram do gênero feminino ( $\mathrm{n}=483,40,6 \%$ da amostra total). 
Foi observado que 475 (39,9\%) participantes nos ensaios selecionados eram do gênero masculino e 233 participantes (19,6\% da amostragem total de 1.191 participantes) não foram especificados pelos autores nos ensaios clínicos selecionados. Observou-se ainda que o tempo de regeneração é um fator de suma relevância no campo de estudo com tais fármacos visando o processo de ganho ósseo. Quanto a isso, os estudos selecionados tinham uma duração mínima de 3 meses e máxima de 9 meses de avaliação pelos autores, sem eventos adversos relatados em ambos os grupos randomizados durante o estudo com as estatinas em associação ou não a demais fármacos.

\section{Discussão}

O presente estudo de revisão integrativa buscou descrever por meio de ensaios clínicos publicados na literatura científica nos últimos 10 anos os efeitos das estatinas no processo de ganho ósseo após procedimentos odontológicos. Por meio dos achados, pode-se evidenciar, na grande maioria dos ensaios selecionados, efeitos benéficos da terapia, uma vez que os resultados mostraram preenchimento ósseo significativo nos grupos testados com tais fármacos da classe de hipolipemiantes.

Com isso, as estatinas, inibidoras da 3-hidroxi-2-metil-glutaril coenzima A redutase, são descritas na farmacologia como um grupo de drogas hipolipemiantes amplamente utilizadas nas ciências da saúde para prevenir ações cardiovasculares no corpo humano (Shirke et al., 2019; Chatterjee et al., 2019). Esses fármacos possuem respostas promissoras em tratamentos médicos, promovendo efeitos benéficos em sua aplicabilidade clínica (Sousa et al., 2017; Pereira et al., 2016; Gouda et al., 2021; Issa et al., 2020; Degala \& Bathija, 2018; Garg \& Pradeep, 2017)

Atualmente, o uso das estatinas vem ganhando impulso no manejo das doenças periodontais devido aos seus efeitos anti-inflamatórios e imunomoduladores (Shirke et al., 2019; Martande et al., 2017; N-Prianka et al., 2017; Pradeep et al., 2012; Chatterjee et al., 2019). Além desses efeitos, as estatinas têm vários benefícios adicionais, incluindo a formação de proteína morfogênica óssea (BMP-2) (Sousa et al., 2017) e supressão da osteoclastogênese (Shirke et al., 2019). Esses fármacos são utilizados em diversos campos das ciências da saúde, sendo a Odontologia uma das áreas que avaliam o efeito promissor desses hipolipemiantes (Pereira et al., 2016; Yaghobee et al., 2020; Shirke et al., 2019).

Diante do exposto, na cirurgia oral, por exemplo, medicamentos que visem o controle de dor, edema, trismo são fundamentais na redução dos efeitos fisiológicos do procedimento invasivo (Chauhan et al., 2018), assim diversos medicamentos são prescritos, dentre eles os hipolipemiantes, como forma de promover a atividade osteoblástica e estimular a regeneração tecidual óssea (Degala \& Bathija, 2018). Com isso, observou-se que uma das alternativas presentes em grandes números em estudos incluídos foi a sinvastatina (Pereira et al., 2017; Gouda et al., 2021; Issa et al., 2020; Yaghobee et al., 2020; Pooja et al., 2019; Degala \& Bathija, 2018; Martande et al., 2017; N-Prianka et al., 2017; Pradeep et al., 2012)

Quanto ao seu método de uso, este medicamento pode ser usado por via oral para tratar a hipercolesterolemia e hiperlipidemia, possuindo ações antirreabsortivas e efeitos anabólicos nos ossos (Yagbobee et al., 2018; Yagbobee et al., 2020; Shirke et al., 2019; Sousa et al., 2017). Para Degala \& Bathija (2018), a sinvastatina pode promover atividade osteoblástica auxiliando na diferenciação dos osteoblastos induzida pela proteína morfogenética óssea (PBM-2). Sua observação foi justificada uma vez que seu ensaio clínico com a utilização do medicamento a 10 miligrama $(\mathrm{mg})$ por meio de aplicação local em cirurgia de terceiros molares demonstrou valores significativos em termos de regeneração óssea em um curto prazo de avaliação, sendo considerado uma alternativa a baixo custo que demonstra uma boa aplicabilidade clínica no processo de osteoindução.

Com dados divergentes em um mesmo campo de estudo e fármaco avaliado, o estudo de Yaghobee et al. (2020) diferem dos resultados obtidos por Degala e Bathija (2018) em termos de aplicabilidade do medicamento, uma vez que a sinvastatina não evidenciou dados que sugerem a sua aplicabilidade clínica nesses processos específicos, não sendo encontradas diferenças na quantidade de osso neoformado e de partículas residuais entre os grupos de material ósseo bovino 
sozinho ou em associação com a sinvastatina. Diferentemente aos dados de Yaghobee et al. (2020) e similar aos resultados de Degala e Bathija (2018), Pradeep et al. (2012) investigaram a eficácia de $1,2 \mathrm{mg}$ de sinvastatina como um sistema de distribuição local de drogas como forma de complemento para raspagem e alisamento radicular para o tratamento de defeitos de furca de classe II. Como resultado, a avaliação deste método demonstrou um efeito positivo em sua aplicabilidade clínica, promovendo aumento na formação óssea por meio de uma alternativa confortável e flexível ao paciente.

Além da cirurgia oral e periodontia, outros campos da odontologia têm avaliado a eficácia do medicamento, sendo um deles a implantodontia. Para Gouda et al. (2021) o implante dentário é um método de tratamento eficaz para reabilitação oral. Com isso, os autores compararam as mudanças na altura e porcentagem de Puerarina contra a utilização de sinvastatina como materiais osteocondutores em 20 pacientes que realizaram aumento de seio facial. Os autores destacam que a puerarina e sinvastatina são materiais de enxerto ósseo clinicamente benéficos na odontologia, principalmente se tratando da região avaliada no estudo.

No campo da periodontia, Preadeep et al. (2016a), compararam a eficácia de 1,2\% de rosuvastatina e 1,2\% de atorvastatina gel de entrega local de drogas para o tratamento de defeitos intraósseos em pacientes com periodontite crônica. Em suma, as melhorias nestes parâmetros foram significativamente maiores com a rosuvastatina de entrega local do que atorvastatina ou géis de placebo em um período de avaliação de 6 e 9 meses. Em um período de observação igual, o estudo de Issa et al. (2020) obteve resultados que demonstraram, assim como os autores que o uso da sinvastatina pode melhorar os parâmetros clínicos de tecidos duros, visto que observaram que o tratamento da superfície da raiz com ácido etilenodiamino tetra-acético pode aumentar a disponibilidade do fármaco sinvastatina na área em que ocorre o defeito ósseo.

Seus achados foram similares aos de Martande et al. (2016) em termos de comparação da atorvastatina em uma mesma porcentagem de solução e tempo de avaliação, no qual também evidenciou que o fármaco sozinho não obteve sucesso no aumento do potencial regenerativo de fibrina rica em plaquetas em doenças periodontais em um mesmo período de avaliação. Seu estudo, também obteve desfecho similar ao de Garg e Preadeep (2017) no qual a atorvastatina também obteve valores reduzidos em comparação ao grupo tratado com o fármaco rosuvastatina em um período igual ao dos autores citados.

Em contrapartida, Martande et al. (2016) e Shirke et al. (2019) relataram que a atorvastatina em associação a raspagem e alisamento radicular pode promover uma resposta regenerativa promissora no tratamento de periodontite crônica. Os autores complementam citando que essa terapia pode ser considerada promissora e não invasiva, podendo promover ainda uma melhora nos parâmetros clínicos dos grupos avaliados. Divergentes aos achados clínicos de Preadeep et al. (2016a), o estudo de Kumari et al. (2016) com pacientes com diagnóstico de diabetes do tipo II (DM2) demonstrou que houve dados significativos em termos estatísticos na aplicação de atorvastatina em gel. Assim, o medicamento foi considerado pelos autores como eficaz no tratamento de defeitos intraósseos em pacientes com DM2, visto que através do exame de radiografia o grupo tratado com o fármaco evidenciou redução de profundidade no defeito ósseo em um período de 9 meses. Seu desfecho foi similar ao de Kumarini et al. (2017), demonstrando que atorvastatina também se demonstrou promissora no reparo ósseo de periodontite crônica.

Preadeep et al. (2016b) realizaram um novo estudo no mesmo ano e com um mesmo número amostral de participantes ( $\mathrm{n}=90$ ) no qual compararam a eficácia do desbridamento com retalho aberto com ou sem fibrina rica em plaquetas (PRF) ou PRF com gel de rosuvastatina também a 1,2\% no tratamento de defeitos intraósseos. O estudo obteve resultados similares ao anterior no que se refere ao índice de redução de defeito nos três grupos avaliativos. Neste caso em questão, a inclusão de 1,2\% de gel de rosuvastatina ao PRF resultou em reduções de profundidade de sondagem e tratamento de defeitos intraósseos ao longo de 9 meses em comparação com outros grupos testados.

Pankaj et al. (2018) obteve resultados similares ao de Preadeep et al. (2016b) em termos de uso do gel de rosuvastatina. Os autores também obtiveram uma amostra total de 90 participantes. Os pesquisadores observaram as reduções 
médias em profundidade de sondagem, ganho de nível de adesão clínica e porcentagem de preenchimento ósseo foram maiores nos grupos rosuvostatina e metformina do que no grupo placebo. Como conclusão do ensaio, o uso de gel de rosuvostatina a 1,2\% demonstrou superioridade em níveis de significância em seu uso.

Nessa perspectiva, a periodontite é descrita na literatura científica como uma doença inflamatória dos tecidos de sustentação dos dentes, causada por um grupo de microrganismos específicos, resultando na reabsorção óssea criando defeitos ósseos, que podem causar a perda do dente (Pooja et al., 2019). Vários fármacos da classe estatinas, por exemplo, sinvastatina, lovastatina e pravastatina modulam a formação óssea (Pankaj et al., 2018). O início e a progressão da periodontite são resultados da resposta inflamatória imune do hospedeiro aos patógenos orais (Pooja et al., 2019). Com isso, vários agentes farmacológicos estão sendo administrados localmente, para melhorar a saúde periodontal (Chatterjee et al. 2019; Martande et al., 2016; Shirke et al., 2019).

Nesse contexto, Chatterjee et al. (2019) verificaram que na comparação entre grupos em seu ensaio, houve ganho significativo em nível de adesão clínica no grupo tratado com gel de rosuvostatina a 1,2\%, raspagem e desbridamento da raiz, além disso, um preenchimento ósseo significativo. Nenhum efeito adverso foi observado nesta avaliação. Os autores concluem que o gel de rosuvostatina a 1,2\% quando administrado localmente pode melhorar os parâmetros clínicos periodontais, bem como elevar o nível de preenchimento ósseo.

Na odontologia, a necessidade de uma regeneração óssea significativa em procedimentos da profissão resultou na busca de materiais para prevenir a reabsorção do osso alveolar, além de induzir a formação de novo osso (Yaghobee et al., 2020). Para Preadeep et al. (2016c) o material de enxerto ósseo de hidroxiapatita porosa possui em sua composição uma resposta clinicamente satisfatória quando usado para preencher defeitos intraósseos periodontais. Em seu estudo, o tratamento de defeitos de furca com 1,2 mg de gel de rosuvastatina combinado com fibrina rica em plaquetas autólogo e enxerto ósseo de hidroxiapatita porosa no qual resultaram em melhorias significativas dos parâmetros clínicos e radiográficos em processos regenerativos ósseos.

As estatinas afetam de maneira direta as células osteoclastos, que dependem da inibição da formação de intermediários necessários para adição de moléculas de origem hidrófobas em uma proteína que inibem a atividade osteoclástica (Shirke et al., 2019). A sinvastatina, por exemplo, auxilia na regeneração óssea e tem um efeito anti-inflamatório quando administrada ou aplicada localmente (Pradeep et al., 2012). É caracterizada como uma estatina hidrofílica sintética de segunda geração, contendo o enxofre, com potentes mecanismos de ação anti-inflamatória (Pradeep et al., 2016c), diferenciação óssea e incluindo ainda a prevenção de reabsorção óssea (Yaghobee et al., 2020).

Sobre isso, Pooja et al. (2019), assim como Preadeep, et al. (2016a) e Martande et al. (2016) investigaram a eficácia clínica de estatinas como administração local de droga como um adjunto a raspagem e alisamento radicular no tratamento da periodontite crônica e regeneração óssea. O fármaco de escolha foi a sinvastatina a 1,2\%. O estudo durou 6 meses e considerado estatisticamente significativo com redução da periodontite, e assim como Chatterjee et al. (2019), maior ganho de nível de adesão clínica e maior preenchimento de defeito intraósseo foi observado em locais tratados com a raspagem, alisamento radicular e sinvastatina.

Similar ao estudo de Pooja et al. (2019), Pryanca et al. (2017) investigaram a eficácia da sinvastatina com a mesma posologia e metodologia de entrega de drogas local no tratamento da periodontite agressiva em defeitos intraósseos. Como resultados similares, também houve a diminuição média da periodontite em um mesmo período. Quanto ao nível de significância do estudo, houve uma média significativamente maior de preenchimento ósseo no grupo tratado com raspagem e alisamento radicular e sinvastatina. Para os autores, assim como nos achados de Pooja et al. (2019), a sinvastatina fornecida localmente promove um método confortável para melhorar os parâmetros clínicos e gerar a formação óssea. 
Com base nos dados coletados por meio de estudos presentes na literatura atual, nota-se que tais medicamentos em sua grande maioria possuem uma aplicabilidade promissora em termos de regeneração óssea no tratamento de doenças e intervenções da cavidade (Chatterjee et al., 2019; Degala \& Bathija, 2018; Kumari et al., 2016). Dentre os estudos incluídos, tais avaliações demonstram por meio de amostras reduzidas um efeito benéfico das estatinas no processo de regeneração óssea (Yaghobee et al., 2020), sugerindo ainda que os fármacos, principalmente a sinvastatina, pode ser uma alternativa de baixo custo (Degala \& Bathija, 2018), com capacidade de acelerar o processo de regeneração alveolar, o que pode sugerir novas terapias em diversas especialidades da Odontologia com o fármaco.

\section{Considerações Finais}

A maioria dos estudos avaliados sugerem o uso de fármacos da classe de hipolipemiantes como uma terapia promissora em cicatrização alveolar, principalmente quando administrados localmente, promovendo respostas positivas no processo de regeneração óssea. Com base nessa informação, este estudo pode contribuir para o conhecimento de cirurgiõesdentistas sobre novas terapias medicamentosas no processo de ganho ósseo em diversos procedimentos da profissão, tornandose uma alternativa promissora em uma Odontologia regenerativa.

Contudo, este estudo de revisão apresentou algumas limitações quanto a aplicabilidade dos medicamentos analisados, visto que em alguns estudos as dosagens dos fármacos dessa categoria não demonstraram diferenças significativas no processo de regeneração óssea em sua aplicação quando em comparação aos demais grupos avaliados, além de evidenciar amostras reduzidas e tempo clínico de avaliação dos ensaios consideravelmente baixos, o que sugere-se que mais estudos com desenho de ensaios clínicos randomizados sejam realizados com o objetivo de elucidar melhor a dosagem e associação farmacológica para determinados procedimentos na Odontologia, visando promover um melhor tratamento medicamentoso a esses pacientes.

\section{Referências}

Anbinder, A. L., Quirino, M. R. S., \& Rocha, R. F. (2006). As estatinas e o tecido ósseo: revisão da literatura. Revista de Odontologia da UNESP, 35(4), 239246.

Chatterjee, D., Kapoor, A., Vijay, S., Sobti, G., Kara, D. \& Thanvi, J. (2019). Efficacy of Locally Administered 1.2\% Rosuvastatin Gel in Patients with Periodontitis: a randomized placebo controlled clinical trial. European Journal Of Dentistry, 13(1), 029-035. https://dx.doi.org/10.1055\%2Fs-0039-1688522.

Chauhan, A. S., \& Maria, A., Managutti, A. (2014). Efficacy of Simvastatin in Bone Regeneration After Surgical Removal of Mandibular Third Molars: a clinical pilot study. Journal Of Maxillofacial And Oral Surgery, 14(3), 578-585. https://doi.org/10.1007/s12663-014-0697-6.

Cláudio, M., Lemos, C., Faverani, L., Santiago-Júnior, J., \& Pellizzer, E. (2017). Efeitos das estatinas associadas à raspagem e alisamento radicular no tratamento da periodontite crônica. Revisão sistemática e metanálise. Archives of Health Investigation, 5. Recuperado de https://archhealthinvestigation.com.br/ArcHI/article/view/2035.

Degala, S., \& Bathija, N. A. (2018). Evaluation of the Efficacy of Simvastatin in Bone Regeneration after Surgical Removal of Bilaterally Impacted Third Molars-A Split-Mouth Randomized Clinical Trial. Journal Of Oral And Maxillofacial Surgery, 76(9), 1847-1858. https://doi.org/10.1016/j.joms.2018.04.035.

Ercole, F. F., Melo, L. S., \& Alcoforado, C. L. G. C. (2014). Revisão integrativa versus revisão sistemática. Revista Mineira de Enfermagem, 18(1), 9-12. http://www.dx.doi.org/10.5935/1415-2762.20140001.

Galvão, T. F., Pansani, T. S. A., \& Harrad, D. (2015). Principais itens para relatar Revisões sistemáticas e Meta-análises: a recomendação prisma. Epidemiologia e Serviços de Saúde, 24(2), 335-342. https://doi.org/10.5123/S1679-49742015000200017.

Garg, S., \& Pradeep, A. R. (2017). 1.2\% Rosuvastatin and 1.2\% Atorvastatin Gel Local Drug Delivery and Redelivery in the Treatment of Class II Furcation Defects: a randomized controlled clinical trial. Journal Of Periodontology, 88(3), 259-265. https://doi.org/10.1902/jop.2016.160399.

Gouda, A., Monim, G. A., \& Khashaba, M. A. (2021). Comparative study to evaluate the effect of Puerarin versus simvastatin as osteoconductive material for Maxillary sinus augmentation: a prospective randomized control clinical trial. Egyptian Dental Journal, 67(2), 1089-1101. https://dx.doi.org/10.21608/edj.2021.52633.1398.

Gutierrez, G. E., Lalka, D., Garrett, I. R., Rossini, G., \& Mundy, G. R. (2006). Transdermal application of lovastatin to rats causes profound increases in bone formation and plasma concentrations. Osteoporosis International, 17(7), 1033-1042. https://doi.org/10.1007/s00198-006-0079-0.

Horiuchi, N., \& Maeda, T. (2006). Statins and bone metabolism. Oral Diseases, 12(2), 85-101. https://doi.org/10.1111/j.1601-0825.2005.01172.x. 
Issa, D.R., Abdel-Ghaffar, K. A., Al-Shahat, M. A., Hassan, A. A. Z., Iacono, V. J., \& Gamal, A. Y. (2019). Guided tissue regeneration of intrabony defects with perforated barrier membranes, simvastatin, and EDTA root surface modification: a clinical and biochemical study. Journal Of Periodontal Research, 55(1), 85-95. https://doi.org/10.1111/jre.12692.

Kumari, M., Martande, S. S., Pradeep, A.R., \& Naik, S. B. (2016). Efficacy of Subgingivally Delivered 1.2\% Atorvastatin in the Treatment of Chronic Periodontitis in Patients With Type 2 Diabetes Mellitus: a randomized controlled clinical trial. Journal Of Periodontology, 87(11), 1278-1285. https://doi.org/10.1902/jop.2016.130227.

Martande, S. S., Kumari, M., Pradeep, A.R., Singh, S. P., Suke, D. K., \& Guruprasad, C.N. (2016). Platelet-Rich Fibrin Combined With 1.2\% Atorvastatin for Treatment of Intrabony Defects in Chronic Periodontitis: a randomized controlled clinical trial. Journal Of Periodontology, 87(9), 1039-1046. https://doi.org/10.1902/jop.2016.150306.

Martande, S. S.; Kumari, M., Pradeep, A. R., Singh, S.P., \& Suke, D. K. (2017). Comparative evaluation of efficacy of subgingivally delivered 1.2\% Atorvastatin and $1.2 \%$ Simvastatin in the treatment of intrabony defects in chronic periodontitis: a randomized controlled trial. Journal Of Dental Research, Dental Clinics, Dental Prospects, 11(1), 18-25. https://doi.org/10.15171/joddd.2017.004.

Oliveira, N. M., Edelmuth, S. V. C. L., Aoki, K. S., \& Gaiotto, K. K. F. (2015). Ação das estatinas no tecido ósseo, Rev.Fac.Ciênc.Méd.Sorocaba, 17(2), 54 57.

Oliveira, O. R. G; Martins, S. P. R., Lima, W. G., \& Gomes, M.M. (2017). O uso de proteínas morfogenéticas ósseas (BMP) e pseudoartroses, uma revisão de literatura. Revista Brasileira de Ortopedia, 52(2), 124-140.

Pankaj, D., Sahu, I., Kurian, I. G., \& Pradeep, A. R. (2018). Comparative evaluation of subgingivally delivered $1.2 \%$ rosuvastatin and $1 \%$ metformin gel in treatment of intrabony defects in chronic periodontitis: a randomized controlled clinical trial. Journal Of Periodontology, 89(11), 1318-1325. https://doi.org/10.1002/jper.17-0434.

Pereira, J. M., Semenoff-Segundo, A., Silva, N. F., Borges, A. H., \& Semenoff, T. A. D. V. (2016). Effect of Simvastatin on induced apical periodontitis in rats: a tomographic and biochemical analysis. Revista de Odontologia da Unesp, 45(4), 189-194. https://doi.org/10.1590/1807-2577.23315.

Pooja, A., Arvind, G., Arun, G., Rajveer, S., Deepak, A., \& Ruby, S. (2019). Beneficial Effects of Subginigivally Delivered 1.2\% Simvastatin Gel in the Treatment of Patients with Chronic Peridontits: A Randomized Clinical Trial. Indian Journal, 9(1), 30-35.

Pradeep, A.R., Garg, V., Kanoriya, D., \& Singhal, S. (2016). 1.2\% Rosuvastatin Versus 1.2\% Atorvastatin Gel Local Drug Delivery and Redelivery in Treatment of Intrabony Defects in Chronic Periodontitis: a randomized placebo-controlled clinical trial. Journal Of Periodontology, 87(7), 756-762. https://doi.org/10.1902/jop.2016.150706.

Pradeep, A.R., Garg, V., Kanoriya, D., \& Singhal, S. (2016). Platelet-Rich Fibrin With 1.2\% Rosuvastatin for Treatment of Intrabony Defects in Chronic Periodontitis: a randomized controlled clinical trial. Journal Of Periodontology, 87(12), 1468-1473. https://doi.org/10.1902/jop.2016.160015.

Pradeep, A.R., Karvekar, S., Nagpal, K., Patnaik, K., Raju, A., \& Singh, P. (2016). Rosuvastatin $1.2 \mathrm{mg}$ In Situ Gel Combined With 1: 1 mixture of autologous platelet-rich fibrin and porous hydroxyapatite bone graft in surgical treatment of mandibular class ii furcation defects. Journal Of Periodontology, 87(1), 5-13. https://doi.org/10.1902/jop.2015.150131.

Pradeep, A.R., Priyanka, N., Kalra, N., Naik, S. B., Singh, S. P., \& Martande, S. (2012). Clinical Efficacy of Subgingivally Delivered 1.2-mg Simvastatin in the Treatment of Individuals With Class II Furcation Defects: a randomized controlled clinical trial. Journal Of Periodontology, 83(12), 1472-1479. https://doi.org/10.1902/jop.2012.110716.

Priyanka, N. A A., Saquib., S., Malgaonkar, N., Kudyar, N., Gupta, A., Kalra, N., \& Pradeep, A. R. (2017). Clinical Efficacy of Subgingivally Delivered 1.2 mg Simvastatin in the Treatment of Patients with Aggressive Periodontitis: a randomized controlled clinical trial. The International Journal Of Periodontics \& Restorative Dentistry, 37(2), 135-141. https://doi.org/10.11607/prd.2936.

Rao, N. S., Pradeep, A. R., Bajaj, P., Kumari, M., \& Naik, S. B. (2013). Simvastatin local drug delivery in smokers with chronic periodontitis: a randomized controlled clinical trial. Australian Dental Journal, 58(2), 156-162. https://doi.org/10.1111/adj.12042.

Santos, C.M.C., Pimenta, C.A.M., \& Nobre, M. R. C. (2007). A estratégia PICO para a construção da pergunta de pesquisa e busca de evidências. Revista Latino-Americana de Enfermagem, 15(1), p. 508-511. https://doi.org/10.1590/S0104-11692007000300023.

Shirke, P. Y., Kolte, A. P., Kolte, R. A., \& Bawanakar, P. V. (2019). Evaluation of the clinical efficacy of $1.2 \%$ atorvastatin by CBCT in the treatment of periodontal intrabony defects: a randomized controlled clinical trial. Journal Of Dental Research, Dental Clinics, Dental Prospects, 13(3), 183-191. https://doi.org/10.15171/joddd.2019.029.

Silva, R.M., Neto, F. C. P., \& Bertoncello, D. (2008). Efeitos da sinvastatina sobre propriedades biomecânicas de ossos de ratas ovariectomizadas, Revista Médica de Minas Gerais, 18(1), 31-36.

Sousa, D. N., Ferreira, V. M., Souza, J. A. C., Stefani, C. M., \& Roriz, V. M. (2017). Efeito tópico da sinvastatina no metabolismo ósseo: revisão crítica da literatura. Rev Odontol Bras Central, 26(77), 1-8. https://doi.org/10.36065/robrac.v26i77.1136.

Souza, E. Q. M., Swerts, A. A., Bruzadelli, S. R., Brigagão, M. R. P. L., Lima, D. C., \& Fernandes, L. A. (2016). Efeitos da Sinvastatina como coadjuvante no tratamento da doença periodontal induzida em ratos. Rev. Bras. Odontol., 73(1), 20-23.

Souza, M. T., Silva, M. D., \& Carvalho, R. (2010). Revisão integrativa: o que é e como fazer. Einstein, 8(1), 102-106, 2010. http://dx.doi.org/10.1590/s1679$45082010 \mathrm{rw} 1134$.

Stillwell, S. B., Fineout-Overholt, E., Melnyk, B.M., \& Williamson, K. M. (2010). Evidence-Based Practice, Step by Step: searching for the evidence. Ajn, American Journal Of Nursing, 110(5), 41-47. https://doi.org/10.1097/01.naj.0000372071.24134.7e. 
Research, Society and Development, v. 11, n. 2, e51911226044, 2022

(CC BY 4.0) | ISSN 2525-3409 | DOI: http://dx.doi.org/10.33448/rsd-v11i2.26044

Yaghobee, S., Ghahroudi, A. A. R.R., Khorsand, A., Mahmoudi, S., \& Rafiei, S. C. (2018). Radiographic Comparison of Bovine Bone Substitute Alone Versus Bovine Bone Substitute and Simvastatin for Human Maxillary Sinus Augmentation. J Dent (Tehran), 15(1), $20-29$.

Yaghobee, S., Panjnoush, M., Rafiei, S. C., Shakib, P. A., Mahmoodi, S., Rasouli-Ghahroudi, A. A. R., \& Poursafar, F. (2020). Effect of Simvastatin on Bone Regeneration: a histologic and histomorphometric analysis. Journal Of Oral And Maxillofacial Surgery, 78(6), 927-934.

zhttps://doi.org/10.1016/j.joms.2020.01.016 